\title{
Fibronectin modulates thymocyte-thymic epithelial cell interactions following Trypanosoma cruzi infection
}

\author{
Désio Aurélio Farias-de-Oliveira, Vinícius Cotta-de-Almeida, Déa Maria S Villa-Verde/ \\ Ingo Riederer, Juliana de Meis, Wilson Savino
}

Laboratório de Pesquisas sobre o Timo, Instituto Oswaldo Cruz-Fiocruz, Rio de Janeiro, RJ, Brasil

\begin{abstract}
Developing thymocytes interact with thymic epithelial cells (TECs) through cell-cell interactions, TEC-derived secretory moieties and extracellular matrix (ECM)-mediated interactions. These physiological interactions are crucial for normal thymocyte differentiation, but can be disrupted in pathological situations. Indeed, there is severe thymic atrophy in animals acutely infected with Trypanosoma cruzi due to $C D 4^{+} C D 8^{+}$thymocyte depletion secondary to caspase-mediated apoptosis, together with changes in ECM deposition and thymocyte migration. We studied an in vitro model of TEC infection by T. cruzi and found that infected TEC cultures show a reduced number of cells, which was likely associated with decreased proliferative capacity, but not with increased cell death, as demonstrated by bromodeoxyuridine and annexin-V labelling. The infected TEC cultures exhibited increased expression of fibronectin (FN), laminin (LM) and type IV collagen. Importantly, treatment with $F N$ increased the relative number of infected cells, whereas treatment with anti-FN or anti-LM antibodies resulted in lower infection rates. Consistent with these data, we observed increased thymocyte adhesion to infected TEC cultures. Overall, these results suggest that ECM molecules, particularly FN, facilitate infection of the thymic epithelium and that the consequent enhancement of ECM expression might be associated with changes in TEC-thymocyte interactions.
\end{abstract}

Key words: Trypanosoma cruzi - thymic epithelial cells - thymocytes - fibronectin - laminin

The thymus is the primary lymphoid organ in which bone marrow-derived $\mathrm{T}$ cell precursors undergo differentiation, leading to the migration of mature, positively selected $\mathrm{T}$ lymphocytes to the T-dependent areas of secondary lymphoid organs. $\mathrm{T}$ cell differentiation begins with the initiation of T cell receptor (TCR) rearrangements by immature $\mathrm{CD} 4{ }^{-} \mathrm{CD} 8$ - thymocytes and progresses with the generation of $\mathrm{CD}^{+} \mathrm{CD}^{+}$cells that acquire TCR expression. Thymocytes that do not undergo a productive TCR gene rearrangement die by apoptosis, whereas cells expressing productive TCRs interact with the self-peptides presented by major histocompatibility complex molecules that are expressed on thymic-microenvironment cells. This interaction determines thymocyte fate through positive or negative selection and the positively selected thymocytes will survive and become mature $\mathrm{CD} 4^{+}$or $\mathrm{CD} 8^{+}$ single-positive $\mathrm{T}$ cells. These developmental steps lead to thymocyte interactions with several non-lymphoid thymic-microenvironment cells, most of which are thymic epithelial cells (TECs), through cell-cell interactions, TEC-derived secretory moieties and extracellular matrix (ECM)-mediated interactions (Savino et al. 2002, 2004, Ciofani \& Zúñiga-Pflücker 2007).

These physiological interactions can be disrupted in certain pathological situations. In particular, the thy-

doi: 10.1590/0074-0276130071

Financial support: IOC/FIOCRUZ, FAPERJ, CNPq, CAPES

+ Corresponding author:dvv@ioc.fiocruz.br

Received 7 February 2013

Accepted 16 August 2013 mus can be considered as a target organ in acute infectious diseases (Savino 2006). Accordingly, there is severe thymic atrophy in animals acutely infected with Trypanosoma cruzi, resulting from $\mathrm{CD} 4^{+} \mathrm{CD} 8^{+}$thymocyte depletion, which is essentially due to caspase-mediated apoptosis (Farias-de-Oliveira et al. 2013) and, to a lesser extent, the abnormal export of immature thymocytes from the thymus. Importantly, we have demonstrated that a portion of these immature thymocytes escaping to the peripheral lymphoid organs in infected animals express "prohibited" V $\beta$ segments of the TCR and adopt the phenotype of activated T cells (Cottade-Almeida et al. 2003, Mendes-da-Cruz et al. 2003, Morrot et al. 2011).

Along with the marked thymocyte depletion observed in acutely infected animals, we found an enhancement in the deposition of ECM proteins in the thymic microenvironment and increased levels of ECM receptor membrane expression in developing thymocytes (Savino 2006).

ECM proteins, such as fibronectin (FN), laminin (LM) and type IV collagen, have been shown to actively contribute to the interaction of developing $\mathrm{T}$ cells with the thymic epithelium during the intrathymic migration of thymocytes (Savino et al. 2002, 2004).

Nevertheless, it remains unclear to what extent the infection itself triggers these ECM changes in the thymic microenvironment. In this work, we analysed the influence of $T$. cruzi infection on TECs and TEC-thymocyte interactions.

\section{MATERIALS AND METHODS}

Animals - Male BALB/c mice, aged four-five weeks, were obtained from the animal facility of the Oswaldo Cruz Foundation (Fiocruz) (Rio de Janeiro, Brazil). 
Chemicals and antibodies - RPMI-1640 culture medium, bovine serum albumin, penicillin, streptomycin, 2-mercaptoethanol, L-glutamine, trypsin, o-phenylenediamine (OPD), 3-amine-9-ethyl-carbazole and 4,6-diamidino-2-phenylindole (DAPI) were purchased from Sigma (St. Louis, USA). Perhydrol, Giemsa, Tween-20 and formaldehyde were purchased from Merck (Rio de Janeiro, Brazil). Foetal calf serum (FCS) was obtained from Cultilab Products (Campinas, Brazil) and the streptavidin-biotinylated horseradish-peroxidase complex was provided by Amersham Int (Buckinghamshire, UK). Recombinant FN, LM and ethylenediaminetetraacetic acid (EDTA) were purchased from Gibco/BRL (Gaithersburg, USA) and the bromodeoxyuridine (BrdU) kit was acquired from Pharmingen/Becton Dickinson (San Diego, USA).

Rabbit polyclonal antibodies against FN, LM or type IV collagen were purchased from Novotec (Saint Martin La-Garenne, France) and labelling was revealed with a rhodamine-coupled goat anti-rabbit secondary antibody (Biosys, Compiègne, France). Anti-VLA-5 PE (anti-CD49e), anti-VLA-6 PE (anti-CD49f), anti-CD3fluorescein isothiocyanate (FITC), anti-CD4-PE and anti-CD8-CyChrome were obtained from Pharmingen/ Becton Dickinson. Immune serum directed against $T$. cruzi was obtained from chronically infected mice in our laboratory and labelling was detected with a rhodaminelabelled rabbit anti-mouse IgG (Biosys).

Cell cultures - The TEC line IT-76M1 was originally developed from a primary culture of BALB/c thymic stromal cells and was kindly provided by Dr T Itoh (Tohuku University Medical School, Sendai, Japan) (Cirne-Lima et al. 1993). The ability of these cells to produce ECM proteins, including LM, FN and type IV collagen, was previously demonstrated (Savino et al. 1986, Lannes-Vieira et al. 1991). Thymic nurse cells (TNCs), which form lymphoepithelial complexes that are composed of one TEC that harbours two-200 differentiating thymocytes, were isolated as previously described (Wekerle et al. 1980, Villa-Verde et al. 1993) and then plated in tissue culture dishes in complete RPMI medium for $60 \mathrm{~h}$ prior to infection. The Vero cell line was applied for the in vitro growth of T. cruzi (Liebhaber et al. 1967).

All cell cultures were maintained under the same conditions: RPMI-1640 medium, $\mathrm{pH} 7.2$, supplemented with $10 \%$ FCS, $100 \mathrm{IU} / \mathrm{mL}$ penicillin, $100 \mu \mathrm{g} / \mathrm{mL}$ streptomycin, 2-mercaptoethanol $\left(5 \times 10^{-5} \mathrm{M}\right)$ and Lglutamine $(2 \mathrm{mM})$ at $37^{\circ} \mathrm{C}$ in an atmosphere containing 5\% $\mathrm{CO}_{2}$. Semi-confluent TEC cultures were treated with $0.25 \%$ trypsin $/ 0.02 \%$ EDTA in $\mathrm{Ca}^{2+}$ - and $\mathrm{Mg}^{2+}$-free solution for 5-7 min, washed, plated as single-cell suspensions and allowed to grow for the experimental assays described below.

Infection of TEC cultures by T. cruzi - Highly infective trypomastigote forms of the Colombian strain (Federici et al. 1964) were obtained from infected Vero cell cultures. The parasites released after five days by infected cultures were washed with PBS, resuspended in serum-free RPMI and used at a ratio of 20 parasites/TEC. After $6 \mathrm{~h}$ of co-culture, the free parasites were discarded by repeated washing and the TEC/TNC cultures were maintained for an additional $48 \mathrm{~h}$. The cells were fixed in absolute methanol for $7 \mathrm{~min}$ and evaluated by Giemsa staining and immunofluorescence labelling with mouse anti-T. cruzi immune serum or DAPI staining.

ECM-related modulation of T. cruzi infection in TEC cultures - TEC cultures were allowed to grow for $24 \mathrm{~h}$ and were then incubated $\left(30 \mathrm{~min}\right.$ at $\left.37^{\circ} \mathrm{C}\right)$ with $\mathrm{FN}$ or LM $(10 \mu \mathrm{g} / \mathrm{mL})$ or with an anti-LM, anti-FN or antitype IV collagen antibody $(10 \mu \mathrm{g} / \mathrm{mL})$. After extensive washing, the TEC cultures were subjected to infection procedures, followed by culture fixation and parasite labelling, as described.

Detection of ECM components in TEC cultures - The detailed technique used to evaluate the ECM components by immunofluorescence was published elsewhere (Berrih et al. 1985, Lannes-Vieira et al. 1991). Briefly, fixed TEC cultures from eight-well Lab-Tek flasks (Corning, Midland, MI, USA) (initially plated at $5 \times 10^{3}$ cells/chamber) were incubated with anti-LM, anti-FN or anti-type IV collagen antibody for $1 \mathrm{~h}$ at room temperature (RT), followed by washing and further incubation with a rhodamine-labelled secondary antibody. Cultures were double-labelled using DAPI to stain the DNA from both the host cells and intracellular parasites. The slides were mounted in glycerine/PBS $(50 \% \mathrm{v} / \mathrm{v})$ and the double staining was analysed by fluorescence microscopy (Leitz, Orthoplan model, Germany).

In an alternative protocol, ELISA was used to evaluate the expression of ECM ligands. TECs were plated in a 96 -well plate $\left(2.5 \times 10^{3}\right.$ cells/well $)$ and maintained in culture for $24 \mathrm{~h}$ followed by the infection protocol. At $48 \mathrm{~h}$ post-infection, the cell cultures were fixed and incubated with anti-LM, anti-FN or anti-type IV collagen antibody, as defined. The cells were then washed and labelled with a biotinylated goat anti-rabbit secondary antibody followed by a final incubation with the streptavidin-peroxidase complex. The plate was washed with PBS/Tween 20 after each incubation step. The assay for enzymatic activity was developed using OPD in the presence of hydrogen peroxide and stopped with sulphuric acid according to a previously reported protocol (Lannes-Vieira et al. 1991). The results were measured using a 492-nm filter (Titertek Multiskan MCC/340, LabSystems, Finland). The negative control included an unrelated primary antibody that did not generate any significant labelling.

A quantitative evaluation of LM deposition on infected and uninfected TECs was performed by the computational analysis of immunostained cell cultures. Images were acquired using a $40 \mathrm{X}$ objective with a colour video camera mounted on an Olympus BX51 microscope (Olympus UK Ltd, Southern-on-Sea, Essex, UK). A quantitative fluorescence analysis was performed by transforming the specific staining into an eight-bit grey image. The software tool Mean Gray Value (the sum of the grey values of all the pixels in the selection divided by the total number of pixels) was used to quantify LM expression. At least three dishes from each group were used for these quantifications. Three random pictures 
were obtained from each dish and all the cells in these pictures were quantified. The acquired images were analysed with ImageJ software (Rasband, WS ImageJ, US National Institutes of Health, Bethesda, MD, USA) (rsb.info.nih.gov/ij/).

Proliferation and apoptosis of cultured TECs - We evaluated the number of TECs in the cultures by directly counting single-cell suspensions after detaching the cells with $0.25 \%$ trypsin $/ 0.02 \%$ EDTA in $\mathrm{Ca}^{2+}$ - and $\mathrm{Mg}^{2+}$-free solution for $5-7 \mathrm{~min}$ at $37^{\circ} \mathrm{C}$, followed by washing and resuspension in PBS in a Neubauer chamber. Additionally, the proliferative status of the infected and uninfected TEC cultures was analysed using a BrdU incorporation assay $(1 \mu \mathrm{M})$ for $30 \mathrm{~min}$ at $37^{\circ} \mathrm{C}$. The presence of $\mathrm{BrdU}^{+}$ cells was determined by fluorescence microscopy, as previously described (Savino \& Dardenne 1985).

An assessment of TEC apoptosis by annexin-V labelling was performed according to the manufacturer's instructions (Southern Biotech, USA). Briefly, suspensions of the cells present in the supernatant and trypsinised cells from TEC cultures were pooled and incubated with annexin buffer for $20 \mathrm{~min}$ at RT, washed and then stained with FITC-coupled annexin-V. The acquisition and data analysis were performed using the FACSCalibur flow cytometer (Becton Dickinson, San Jose, CA, USA) and CellQuest software.

Membrane expression of ECM receptors on cultured TECs - Single-cell suspensions of TECs were obtained after trypsin treatment as described and subjected to labelling with an FITC-coupled anti-CD49e or anti-CD49f antibody, as previously reported (Ribeiro-Carvalho et al. 2007). After washing, the cells were fixed in $0.5 \%$ formaldehyde and examined by flow cytometry.

Thymocyte-TEC adhesion assay - TECs were allowed to adhere to culture flasks ( 1 x $10^{5}$ cells) and were infected with $T$. cruzi $24 \mathrm{~h}$ later. After extensive washing with PBS, the cultures were maintained for $48 \mathrm{~h}$, as described. The cultures were then incubated with $5 \mathrm{x}$ $10^{6}$ thymocytes, which were obtained by the mechanical disruption of control thymuses, in serum-free RPMI medium for $30 \mathrm{~min}$ at $37^{\circ} \mathrm{C}$ followed by mild shaking (60 rpm) for $30 \mathrm{~min}$ at RT. Floating, non adherent thymocytes were removed by a single gentle washing of the plates with $\mathrm{PBS}$ at $37^{\circ} \mathrm{C}$. The plates were fixed in methanol for $7 \mathrm{~min}$ and stained with Giemsa solution. The number of adhered thymocytes per at least 1,000 TECs were directly counted and the association index (AI) was calculated as follows:

$\mathrm{AI}=\frac{\text { TECs with bound thymocytes }}{\text { total TEC number }} \times \frac{\text { thymocytes bound to TECs }}{\text { total TEC number }} \times 100$

The quantification of adherent thymocytes in the infected or uninfected TEC cultures was performed after extensive PBS washing to detach the thymocytes from the TECs, with subsequent direct counting in a Neubauer chamber.

Statistical analysis - Unpaired Student's $t$ tests or Mann-Whitney $U$ tests were applied for the statistical analyses. Differences between the control and the in- fected groups were considered statistically significant when $\mathrm{p}<0.05$.

Ethics - All the experimental procedures were previously approved by the Ethical Committee for Animal Research of the Fiocruz.

\section{RESULTS}

T. cruzi infection enhances the ECM content of cultured TECs - Confirming our previous results (Da Costa et al. 1991), we initially showed that T. cruzi-TEC interaction (20 parasites/TEC) for $6 \mathrm{~h}$ resulted in the infection of approximately $5 \%$ of the cultured cells, demonstrating that TECs can be infected in vitro.

Considering that $T$. cruzi infection promotes an increase in ECM deposition in the mouse thymus, we confirmed that the content of these proteins also increased in infected TEC cultures, as illustrated in Fig. 1A, B. This increase was observed for the three ECM proteins evaluated, namely, FN, LM and type IV collagen, by qualitative and quantitative immunofluorescence analyses and ELISA.

We then analysed whether the membrane expression of integrin-type ECM receptors was also altered in the infected cultures. As ascertained by the cytofluorometric analysis of surface CD49e (the $\alpha$ chain of the FN receptor VLA-5) and CD49f (the corresponding $\alpha$ chain of the LM receptor VLA-6), no significant difference was observed. Similar values were found for both the relative cell number and the membrane density of each receptor (Fig. 1C).

T. cruzi infection modulates TEC/thymocyte interactions - Because ECM ligands and receptors have been shown to play a role in TEC-thymocyte interactions, we investigated whether $T$. cruzi infection could modulate the degree of thymocyte adhesion to TECs in infected cultures. In fact, we found that infection enhanced the adhesion of thymocytes to TECs, as demonstrated by Giemsa staining and quantified by calculating the AI (Fig. 2A, B). Interestingly, when we analysed the infected cultures by quantifying thymocyte adhesion, comparing the infected and uninfected cells, we found that the highest degree of TEC-thymocyte association was observed when the TECs were parasitised (Fig. 2B, right panel). Lastly, the cytofluorometric profiles of thymocyte subsets defined by the markers CD4 and CD8 that adhered to the infected or uninfected TEC cultures showed no significant differences (Fig. 2C).

ECM ligands are involved in T. cruzi infection of the thymic epithelium - The notion that ECM components are involved in the interaction between different host cells and T. cruzi (Ouaissi et al. 1985, Giordano et al. 1994, Nde et al. 2006) prompted us to investigate whether ECM ligands could also be involved in TEC infection by $T$. cruzi. When we treated TEC cultures with purified $\mathrm{FN}$, the relative number of infected cells was significantly higher when compared to untreated infected TEC cultures; the opposite effect was observed when the TEC cultures were treated with anti-ECM antibodies prior to infection (Fig. 3A). Furthermore, these re- 
sults were confirmed when primary cultures of TNCs previously treated with anti-ECM were infected with the parasite (Fig. 3B). Altogether, these results suggest that ECM proteins are directly involved in TEC-parasite interactions, positively modulating the number of infected epithelial cells.

T. cruzi infection reduces the proliferation of cultured TECs - We lastly evaluated whether T. cruzi infection could modulate other parameters in TEC physiology, such as cell proliferation and death. Direct TEC counting showed a decreased cell number in the infected TEC cultures compared to the controls, suggesting that

A
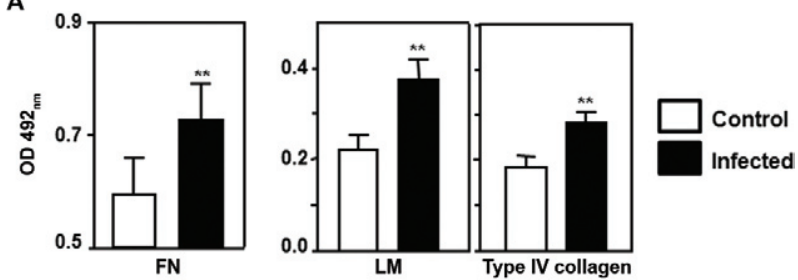

B

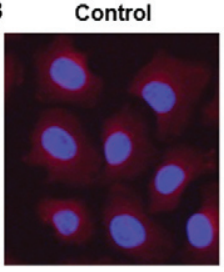

Infected
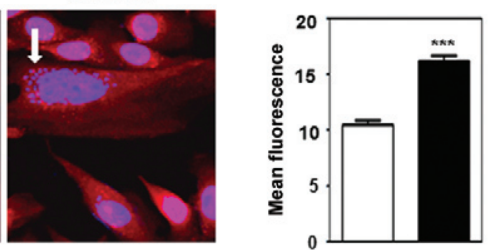

C

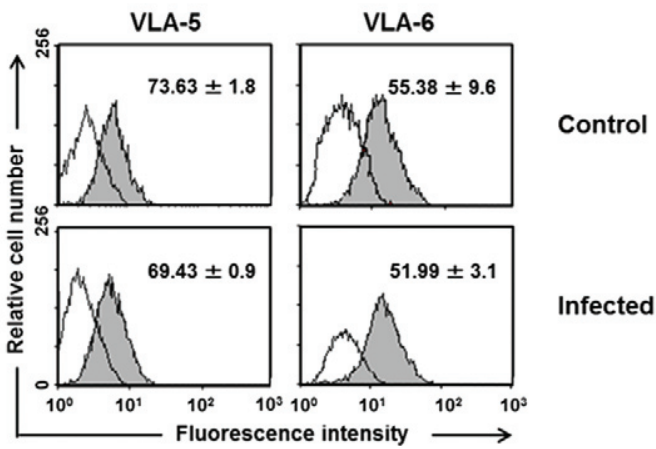

Fig. 1: Trypanosoma cruzi infection enhances extracellular matrix (ECM) contents in growing thymic epithelial cells. A: ELISA data revealing the enhancement of ECM contents in T. cruzi infected thymic epithelial cells (TECs) cultures comprising fibronectin, laminin (LM) and type IV collagen [each bar representing mean \pm standard deviation (SD) of optical density (OD) readings from 12 wells in three independent experiments]; B: representative microscopic fields, respectively from control and infected TEC cultures, immunolabelled for LM detection. Amastigote forms of the parasite (detected with 4,6-diamidino-2-phenylindole staining) are indicated by the arrow. Enhancement of LM contents was further quantitated by morphometry and the mean fluorescence for this molecule was higher in infected cultures, as compared with non-infected controls; C: cytofluorometric analyses of surface CD49e and CD49f: no significant difference was observed between $T$. cruzi infected vs. non-infected TEC cultures. Similar values were found in terms of both relative cell numbers (seen within each square $\pm \mathrm{SD}$ ) and membrane density profiles of each receptor. Micrograph magnification: 400X. **: $\mathrm{p}<0.01$; $* * *: \mathrm{p}<0.001$.
T. cruzi infection might be either inducing cell death or leading to cell cycle arrest. We then evaluated BrdU incorporation by the infected cells and found a decrease in the number of cycling cells in the infected cultures compared to the uninfected cells. No differences in the number of annexin- $\mathrm{V}^{+}$cells were observed between the infected and uninfected TECs. These data are summarised in Fig. 4 and indicate that the T. cruzi infection of TEC cultures inhibited the cells' ability to enter S-phase, resulting in a reduced proliferation rate.
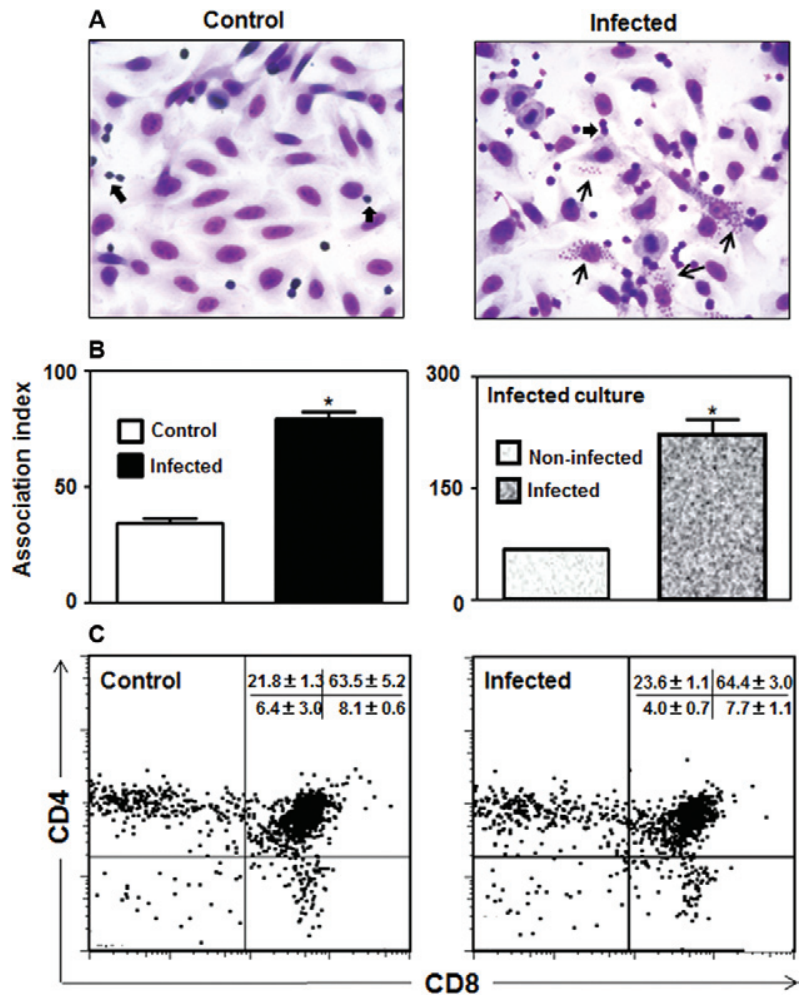

Fig. 2: Trypanosoma cruzi infection modulates adhesion of thymocytes to thymic epithelial cells. A: representative microscopic fields of Giemsa-stained thymic epithelial cells (TECs) cultures showing increased numbers of adhered thymocytes (thick arrows) in TEC from infected cultures, as compared to non-infected controls. This was confirmed by calculating the thymocyte/TEC association index in the TEC cultures as a whole, as shown in B (left side). Further analysis of these infected cultures revealed that infected TEC have even higher association with thymocytes when compared to noninfected cells of the same cultures, as seen in the right side of B. Values represent mean \pm standard deviation of three independent experiments. The phenotype of adhered CD4/CD8 thymocyte subpopulations (C) shows that no preferential adhesion is observed on infected or non-infected TEC. Adhered thymocytes were removed and immunostained with anti-CD3-fluorescein isothiocyanate, antiCD4-PE and anti-CD8-CyChrome at $4^{\circ} \mathrm{C}$ and the percentages of adherent thymocytes in infected or non-infected TEC cultures were defined by flow cytometry, as described in Materials and Methods. Values corresponding to the relative numbers of each CD4/CD8defined subset represent the mean \pm standard error from three independent experiments. Micrograph magnification: 400X. In the infected TEC culture micrograph, thin arrows point to T. cruzi intracellular amastigotes. Asterisk means: $\mathrm{p}<0.05$. 


\section{DISCUSSION}

T. cruzi infection in vivo has been shown to cause alterations in both the lymphoid and the microenvironment compartments of the thymus. Additionally, the modulation of the thymic expression of ECM ligands and receptors in infected mice has been correlated with critical changes in the adhesive and migratory abilities of thymocytes (Cotta-de-Almeida et al. 2003, Mendesda-Cruz et al. 2003, Savino et al. 2004, Mendes-da-Cruz et al. 2006, Silva-Monteiro et al. 2007). In the present study, by employing an in vitro infection approach using cultures of murine TECs, we investigated the role of the ECM in the infection of TECs and analysed whether this infection might interfere with thymocyte-TEC interactions. The T. cruzi-infected TEC cultures showed an enhancement in the content of ECM components, which is in agreement with previous data obtained from different cell types infected with the parasite (Marino et al. 2003, Calvet et al. 2009). Interestingly, we showed that such an increase in ECM proteins was not restricted to the infected cells, as this increase was observed throughout the culturing of infected TECs. These data suggest that parasite antigens released into the culture supernatant may be involved in this effect. Nevertheless, our results show that the incubation of TEC cultures with
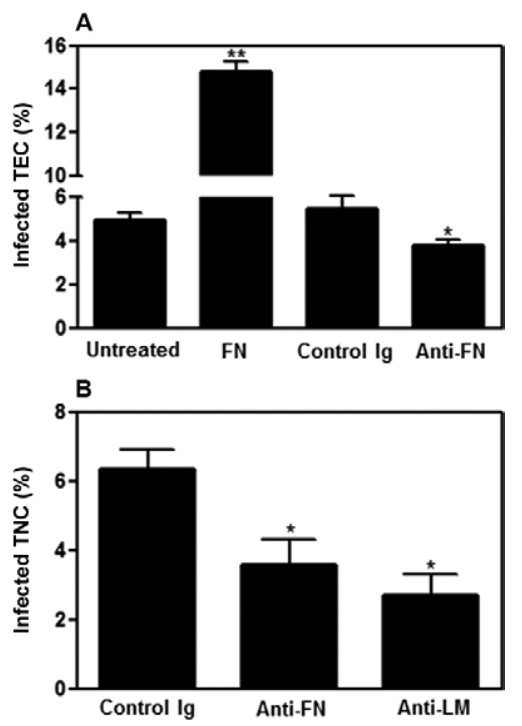

Fig. 3: extracellular matrix ligands are involved in the infectivity of Trypanosoma cruzi towards thymic epithelial cells. A: infection of thymic epithelial cells (TECs) cultures (20 parasites/TEC for $6 \mathrm{~h}$ ) previously treated with fibronectin (FN) $(10 \mu \mathrm{g} / \mathrm{mL}$ for $30 \mathrm{~min})$ resulted in a significant increase in the percentages of infected cells when compared to control untreated cultures. By contrast when TEC were pre-treated with anti-FN antibodies $(10 \mu \mathrm{g} / \mathrm{mL}$ for $30 \mathrm{~min})$, an opposite effect was seen, with a significant reduction of infection rates. Differences were evaluated in three separate experiments by direct counting of 600 cells after indirect immunofluorescence assay for parasite detection; B: effects of anti-FN or anti-laminin (LM) antibodies in the percentage of infection of a primary thymic nurse cell (TNC) culture by T. cruzi are similar to that observed in the TEC line. Values represent mean \pm standard deviation from three independent experiments. $*$ : $\mathrm{p}<0.05 ; *$ : $\mathrm{p}<0.01$. a parasite-conditioned medium did not increase LM expression (data not shown). Another possibility is that other factors secreted by infected cells might cause this widespread modulatory effect on the ECM content. In agreement with this idea, previous studies have demonstrated that Cryptosporidium parvum infection in intestinal epithelial cells modulates interferon- $\gamma$ and tumour necrosis factor- $\alpha$ secretion, followed by an upregulation of the expression of ECM proteins and chemokines (Lacroix et al. 2001, Lacroix-Lamandé et al. 2002). Further experiments are necessary to elucidate this hypothesis in our model.

Because of the modulation of the ECM content by thymic microenvironment cells may result in changes in the patterns of interactions with developing thymocytes, it was logical to speculate that an increase in the ECM content in infected TEC cultures would also have an impact on the interactions of these cells with thymocytes. In fact, we observed an increased adhesion index when the thymocytes were co-cultured with infected TEC cultures. It is likely that these alterations, demonstrated herein in vitro, may occur in vivo and be at least partially involved in the changes in thymocyte migration observed in experimental Chagas disease (Cotta-deAlmeida et al. 2003, Mendes-da-Cruz et al. 2003).
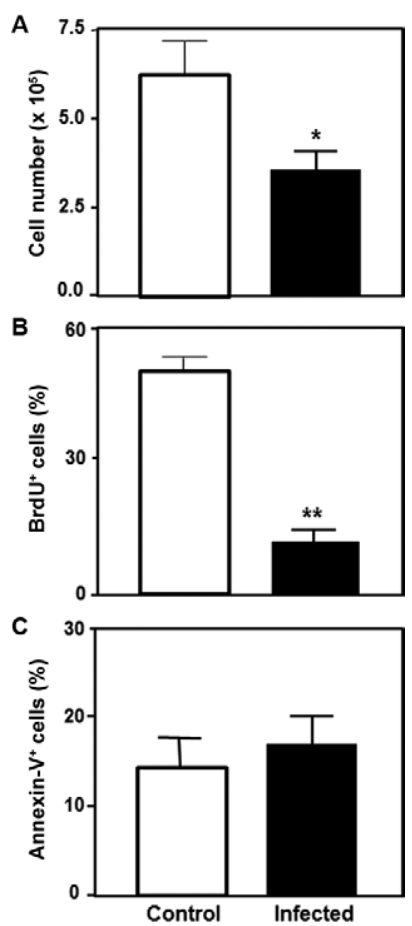

Fig. 4. Trypanosoma cruzi infection decreases the number of replicating thymic epithelial cells. A: the total number of thymic epithelial cells (TECs) is significantly reduced in infected cultures; B, C: bromodeoxyuridine (BrdU) and annexin- $\mathrm{V}$ staining, respectively, decreased cell proliferation and normal frequency of apoptotic cells in infected cultures, as compared to control (non-infected) ones. Data seen in B, C represent the mean + standard deviation of the percentage of labelled TEC from a pool of three independent experiments. *: $\mathrm{p}<$ $0.05 ; * *: \mathrm{p}<0.01$. 
Through a second set of experiments, we provide evidence that $\mathrm{FN}$ favours the entry of $T$. cruzi into cultured TECs. The addition of this ECM protein enhanced the percentage of infected TECs, whereas treatment with the corresponding antibody promoted the opposite effect. Interestingly, the anti-LM antibody treatment also impaired TEC infection. These data were confirmed when a primary TNC culture was treated with anti-ECM antibodies and subjected to infection. From a conceptual point of view, it is conceivable that the increase in the FN content of the infected TEC cultures is directly linked to the success of the invasion of the thymic host cells by the parasite. However, we should consider that other ECM or ECM-related molecules that are increased in TECs during $T$. cruzi infection, such as LM, type IV collagen and galectin-3, participate in the ECM interactome network that is regulated by the parasite to facilitate cell infection (Nde et al. 2006, 2010, 2012, Silva-Monteiro et al. 2007, Cardenas et al. 2010).

Finally, we investigated whether $T$. cruzi infection could directly influence other parameters in TEC physiology, such as cell proliferation and death and found a significant decrease in the number of cells in the infected TEC cultures compared to uninfected cells. This effect was due to a decrease in cell proliferation, as demonstrated by a BrdU incorporation assay, and not to an increase in cell death because no effect of the infection on TEC apoptosis was observed.

In conclusion, T. cruzi infection enhances the production of ECM by the thymic epithelium and takes advantage of this effect to further enhance its infectivity in the thymic microenvironment. Moreover, such an enhancement in the ECM results in an increase in the adhesion of thymocytes to TEC cultures, which may be at the origin of the abnormal thymocyte migration and export observed in infected mice.

\section{REFERENCES}

Berrih S, Savino W, Cohen S 1985. Extracellular matrix of the human thymus: immunofluorescence studies on frozen sections and cultured epithelial cells. J Histochem Cytochem 33: 655-664.

Calvet CM, Oliveira Jr FO, Araujo-Jorge TC, Pereira MC 2009. Regulation of extracellular matrix expression and distribution in Trypanosoma cruzi-infected cardiomyocytes. Int J Med Microbiol 299: 301-312.

Cardenas TC, Johnson CA, Pratap S, Nde PN, Furtak V, Kleshchenko YY, Lima MF, Villalta F 2010. Regulation of the extracellular matrix interactome by Trypanosoma cruzi. Open Parasitol J 4: 72-76.

Ciofani M, Zúñiga-Pflücker JC 2007. The thymus as an inductive site for T lymphopoiesis. Annu Rev Cell Dev Biol 23: 463-493.

Cirne-Lima EO, van Ewijk W, Savino W 1993. Cortical and medullary phenotypes within a mouse thymic epithelial cell line. In Vitro Cell Dev Biol Anim 29A: 443-445.

Cotta-de-Almeida V, Bonomo A, Mendes-da-Cruz DA, Riederer I, De Meis J, Lima-Quaresma KR, Vieira-de-Abreu A, Villa-Verde DM, Savino W 2003. Trypanosoma cruzi infection modulates intrathymic contents of extracellular matrix ligands and receptors and alters thymocyte migration. Eur J Immunol 33: 2439-2448.

Da Costa SC, Calabrese KS, Bauer PG, Savino W, Lagrange PH 1991. Studies of the thymus in Chagas disease: III. Colonization of the thymus and other lymphoid organs of adult and newborn mice by Trypanosoma cruzi. Pathol Biol 39: 91-97.

Farias-de-Oliveira DA, Villa-Verde DMS, Panzenhagen PHN, Santos DS, Berbert LR, Savino W, de Meis J 2013. Caspase-8 and caspase-9 mediate thymocyte apoptosis in Trypanosoma cruzi acutely infected mice. J Leukoc Biol 93: 227-234.

Federici EE, Abelmann WH, Neva FA 1964. Chronic and progressive myocarditis and myositis in C3h mice infected with Trypanosoma cruzi. Am J Trop Med Hyg 13: 272-280.

Giordano R, Chammas R, Veiga SS, Colli W, Alves MJ 1994. An acidic component of the heterogeneous Tc- 85 protein family from the surface of Trypanosoma cruzi is a laminin binding glycoprotein. Mol Biochem Parasitol 65: 85-94.

Lacroix-Lamandé S, Mancassola R, Naciri M, Laurent F 2002. Role of gamma interferon in chemokine expression in the ileum of mice and in a murine intestinal epithelial cell line after Cryptosporidium parvum infection. Infect Immun 70: 2090-2099.

Lacroix S, Mancassola R, Naciri M, Laurent F 2001. Cryptosporidium parvum-specific mucosal immune response in C57BL/6 neonatal and gamma interferon-deficient mice: role of tumor necrosis factor alpha in protection. Infect Immun 69: 1635-1642.

Lannes-Vieira J, van der Meide PH, Savino W 1991. Extracellular matrix components of the mouse thymic microenvironment. II. In vitro modulation of basement membrane proteins by interferon-gamma: relationship with thymic epithelial cell proliferation. Cell Immunol 137: 329-340.

Liebhaber H, Riordan JT, Horstmann DM 1967. Replication of rubella virus in a continuous line of African green monkey kidney cells (Vero). Proc Soc Exp Biol Med 125: 636-643.

Marino AP, Silva AA, Pinho RT, Lannes-Vieira J 2003. Trypanosoma cruzi infection: a continuous invader-host cell cross talk with participation of extracellular matrix and adhesion and chemoattractant molecules. Braz J Med Biol Res 36: 1121-1133.

Mendes-da-Cruz DA, de Meis J, Cotta-de-Almeida V, Savino W 2003. Experimental Trypanosoma cruzi infection alters the shaping of the central and peripheral T-cell repertoire. Microbes Infect 5: 825-832.

Mendes-da-Cruz DA, Silva JS, Cotta-de-Almeida V, Savino W 2006. Altered thymocyte migration during experimental acute Trypanosoma cruzi infection: combined role of fibronectin and the chemokines CXCL12 and CCL4. Eur J Immunol 36: 1486-1493.

Morrot A, Terra-Granado E, Pérez AR, Silva-Barbosa SD, Milićević NM, Farias-de-Oliveira DA, Berbert LR, de Meis J, Takiya CM, Beloscar J, Wang X, Kont V, Peterson P, Bostasso O, Savino W 2011. Chagasic thymic atrophy does not affect negative selection but results in the export of activated $\mathrm{CD} 4^{+} \mathrm{CD}^{+} \mathrm{T}$ cells in severe forms of human disease. PLoS Negl Trop Dis 5: e1268.

Nde PN, Johnson CA, Pratap P, Cardenas TC, Kleshchenko YY, Furtak VA, Simmons KJ, Lima MF, Villalta F 2010. Gene network analysis during early infection of human coronary artery smooth muscle cells by Trypanosoma cruzi and its gp 83 ligand. Chem Biodivers 7: 1051-1064.

Nde PN, Lima MF, Johnson CA, Pratap S, Villalta F 2012. Regulation and use of the extracellular matrix by Trypanosoma cruzi during early infection. Front Immunol 3: 1-10.

Nde PN, Simmons KJ, Kleshchenko YY, Pratap S, Lima MF, Villalta F 2006. Silencing of the laminin gamma-1 gene blocks Trypanosoma cruzi infection. Infect Immun 74: 1643-1648.

Ouaissi MA, Cornette J, Capron A 1985. Trypanosoma cruzi: modulation of parasite-cell interaction by plasma fibronectin. Eur $J$ Immunol 15: 1096-1101. 
Ribeiro-Carvalho MM, Lima-Quaresma KR, Mouco T, Carvalho VF, Mello-Coelho V, Savino W 2007. Triiodothyronine modulates thymocyte migration. Scand J Immunol 66: 17-25.

Savino W 2006. The thymus is a common target organ in infectious diseases. PLoS Pathog 2: e62.

Savino W, Dardenne M 1985. Analysis of thymic epithelial cell proliferation in vitro by combining bromodeoxyuridine and keratin labeling in an immunofluorescence assay. J Immunol Methods 85: 221-226.

Savino W, Itoh T, Imhof BA, Dardenne M 1986. Immunohistochemical studies on the phenotype of murine and human thymic stromal cell lines. Thymus 8: 245-256.

Savino W, Mendes-da-Cruz DA, Silva JS, Dardenne M, Cotta-deAlmeida V 2002. Intrathymic T-cell migration: a combinatorial interplay of extracellular matrix and chemokines? Trends Immunol 23: 305-313
Savino W, Mendes-da-Cruz DA, Smaniotto S, Silva-Monteiro E, Villa-Verde DM 2004. Molecular mechanisms governing thymocyte migration: combined role of chemokines and extracellular matrix. J Leukoc Biol 75: 951-961.

Silva-Monteiro E, Reis Lorenzato L, Kenji Nihei O, Junqueira M, Rabinovich GA, Hsu DK, Liu FT, Savino W, Chammas R, Villa-Verde DM 2007. Altered expression of galectin-3 induces cortical thymocyte depletion and premature exit of immature thymocytes during Trypanosoma cruzi infection. Am J Pathol 170: 546-556.

Villa-Verde DM, de Mello-Coelho V, Farias-de-Oliveira DA, Dardenne M, Savino W 1993. Pleiotropic influence of triiodothyronine on thymus physiology. Endocrinology 133: 867-875.

Wekerle H, Ketelsen UP, Ernst M 1980. Thymic nurse cells. Lymphoepithelial cell complexes in murine thymuses: morphological and serological characterization. J Exp Med 151: 925-944. 